(ง)

\title{
PARAMETRIC IDENTIFICATION OF TRUSS STRU CTURES BY USING TRANSIENT RESPONSE
}

\author{
Pei-Ling Liu and Cheng-Chieh Chen \\ Institute of Applied Mechanics, National Taiwan University, Taipei, Taiwan, \\ Republic of China
}

(Received 21 February 1995, and in final form 20 April 1995)

\begin{abstract}
A method for identifying the element rigidities of a truss is developed. The transient response of the truss is transformed into the frequency domain for use in the identification process. The spectral finite element method is employed to formulate the equilibrium equation of the truss in the frequency domain. The identification problem is then formulated as an optimization program in which the error norm of the equilibrium equation is minimized. The issue of identifiability is addressed. A numerical example is presented to illustrate the proposed method. It is shown the element properties can be obtained successfully. The influence of noise is investigated through an example.
\end{abstract}

(C) 1996 Academic Press Limited

\section{INTRODUCTION}

The field of system identification has gained more and more attention in recent years due to the increasing demand for quality and safety of structures. System identification methods have been employed to improve the mathematical model of the dynamic behaviour of a structure, such as a space station. Then, the improved model is often used to develop a control scheme for the structure. On the other hand, system identification methods have been applied to evaluate the current condition of existing structures. Therefore, they are useful in the safety assessment of structures, especially after unexpected, extraordinary loading.

The literature on system identification is quite extensive. Many researchers have adopted modal data in the identification of structures. Chen and Garba [1] have developed a method to determine the changes in the structure stiffness matrix based on the assumption that the mass matrix does not change with damage. The detection of damage is then attempted by examining the change of the stiffness matrix. Baruch and Bar-Itzhack [2], Berman and Nagy [3], Kabe [4], Kammer [5], and Smith and Beattie [6] have presented an optimal-update identification method which adjusts the mass, damping or stiffness matrix through the solution of a constrained optimization problem. Minas and Inman [7], and Zimmerman and Kaouk [8] have proposed an eigenstructure assignment algorithm to improve the stiffness and damping matrices. Liu [9] has incorporated a finite element formulation in the least squares inversion to determine the axial rigidities of a truss.

Sanayei and Onipede [10], and Sanayei and Scampoli [11] have developed a method for computing stiffness and detecting damage at element level using static test data. Hajela and Soeiro [12] have demonstrated the use of static test data in conjunction with modal data to detect damage in trusses and semi-monocoque structures. Liu and Chian [13] have developed a direct inverse method to identify the axial rigidities of a truss using the static strains of the truss. 
Transient data have also been adopted to identify the dynamic model of a structure. Shinozuka et al. [14] and Agbabian et al. [15] have used the least squares method and time domain response to identify a linear dynamic system. Masri et al. [16, 17] have applied a similar approach to identify the residual and restoring forces of non-linear structures. Lin et al. [18] have employed the Newmark time-integration and least squares method to develop a real time technique to identify the time-varying property matrices of a degrading structure subjected to general dynamic loads. Yun and Shinozuka [19], Hoshiya and Saito [20], and Hoshiya and Maruyama [21] have used the extended Kalman filter to identify linear and nonlinear systems. Mottershead [22] has used recursive, least squares filters on the frequency response function to update the structural parameters.

In this study, an identification method is developed in which the transient response of a truss is used to determine the element properties of the truss. The method is different from the aforementioned transient approaches in that the response in the frequency domain is considered in this method. Furthermore, the element properties rather than the global matrices are obtained. Hence, one can detect the damage in the structure simply by examining the element properties.

In this identification method, the spectral finite element method [23] is used to compute the transient response of the truss. The forward solution is described in the following section.

\section{SPECTRAL FINITE ELEMENT METHOD}

Consider a truss which is composed of uniform prismatic bars. The governing equation of the truss member is

$$
\alpha \partial^{2} u(x, t) / \partial x^{2}=\rho \partial^{2} u(x, t) / \partial t^{2}, \quad 0<x<L,
$$

where $x$ is the position along the length of the element, $t$ is time, $u(x, t)$ is the longitudinal displacement of the element, $\alpha$ is the axial rigidity which is equal to the product of Young's modulus and cross-sectional area, $\rho$ is the mass density per unit length, and $L$ is the length of the element.

By using a discrete Fourier tansform, the general solution of the above equation can be represented by the summation of a finite number of wave trains, i.e.,

$$
u(x, t)=\sum_{n=0}^{N-1} \hat{u}_{n}\left(x, \omega_{n}\right) \mathrm{e}^{\mathrm{i} \omega_{n} t},
$$

where $N$ is the number of wave trains, $\hat{u}_{n}$ are the Fourier coefficients, and $\omega_{n}=2 \pi n / T$ denote the discrete frequencies, $T$ being the duration of the discrete Fourier transform. Substituting the spectral representation into equation (1) and noticing that each $\mathrm{e}^{\mathrm{i} \omega_{n} t}$ term is independent, one can obtain the following simultaneous equations:

$$
\alpha \mathrm{d}^{2} \hat{u}_{n}\left(x, \omega_{n}\right) / \mathrm{d} x^{2}+\omega_{n}^{2} \rho \hat{u}_{n}\left(x, \omega_{n}\right)=0, \quad n=0,1,2, \ldots, N-1 .
$$

Equation (3) has constant coefficients. Hence, it has the obvious solution

$$
\hat{u}_{n}\left(x, \omega_{n}\right)=G_{n} \mathrm{e}^{-\mathrm{i} k_{n} x}+H_{n} \mathrm{e}^{\mathrm{i} k_{n}(L-x)},
$$


where $k_{n}=\omega_{n} \sqrt{\rho / \alpha}$ is called the wavenumber, and $G_{n}$ and $H_{n}$ are the undetermined amplitudes. By substituting $x=0$ and $x=L$ into equation (4), $G_{n}$ and $H_{n}$ can be expressed as

$$
\left[\begin{array}{l}
G_{n}\left(\omega_{n}\right) \\
H_{n}\left(\omega_{n}\right)
\end{array}\right]=\frac{1}{\left(1-\mathrm{e}^{-\mathrm{i} 2 k_{n} L}\right)}\left[\begin{array}{cc}
1 & -\mathrm{e}^{-\mathrm{i} k_{n} L} \\
-\mathrm{e}^{-\mathrm{i} k_{n} L} & 1
\end{array}\right]\left[\begin{array}{l}
\hat{u}_{1}\left(\omega_{n}\right) \\
\hat{u}_{2}\left(\omega_{n}\right)
\end{array}\right]
$$

where $\hat{u}_{1}\left(\omega_{n}\right)=\hat{u}\left(x=0, \omega_{n}\right)$ and $\hat{u}_{2}\left(\omega_{n}\right)=\hat{u}\left(x=L, \omega_{n}\right)$ are the nodal displacements.

Since the axial force of the member is determined by $\alpha \partial u / \partial x$, it is easy to derive

$$
\left[\begin{array}{l}
\hat{F}_{1}\left(\omega_{n}\right) \\
\hat{F}_{2}\left(\omega_{n}\right)
\end{array}\right]=\frac{\alpha}{L} \frac{k_{n} L}{\sin k_{n} L}\left[\begin{array}{cc}
\cos k_{n} L & -1 \\
-1 & \cos k_{n} L
\end{array}\right]\left[\begin{array}{l}
\hat{u}_{1}\left(\omega_{n}\right) \\
\hat{u}_{2}\left(\omega_{n}\right)
\end{array}\right]
$$

where $\hat{F}_{i}$ are the nodal forces. This is the element equilibrium equation.

As in the conventional finite element method, the global equilibrium equation of the truss can be constructed by assembling the element equilibrium equations. The global equation is then condensed by applying the displacement boundary conditions. Let the condensed equation be expressed as

$$
\hat{\mathbf{K}}(\omega) \hat{\mathbf{u}}\left(\omega_{n}\right)=\hat{\mathbf{F}}\left(\omega_{n}\right), \quad n=0,1,2, \ldots, N-1,
$$

where $\hat{\mathbf{K}}$, $\hat{\mathbf{u}}$ and $\hat{\mathbf{F}}$ are the global stiffness matrix, and the nodal displacement and force vectors, respectively. Once the boundary conditions are specified, the nodal displacements corresponding to each frequency can be calculated. Finally, the response in the time domain can be obtained by taking the inverse discrete Fourier transform of the response in the frequency domain.

The spectral finite element method describes wave propagation in a truss element precisely. No interpolation is involved. Hence, the dynamic behavior of a truss element can be obtained accurately irrespective of the element length. Doyle and Farris [24] have demonstrated the validity of the spectral finite element method by an experiment. It was shown that the response obtained by the spectral finite element method agrees well with experimental results. Hence, it is believed that the wave approach can predict the transient response of a truss structure accurately. The coventional finite element method can also be used to calculate the transient response of a truss. However, it uses shape functions to interpolate the displacement in a member. In order to obtain accurate results, each truss member needs to be discretized into sufficiently small elements, and the computation can be expected to be costly. Therefore, the spectral finite element method is more efficient in the transient analysis of truss structures.

\section{PARAMETRIC IDENTIFICATION}

Parametric identification of the truss members can be formulated as the following optimization problem:

$$
\text { minimize } \mathscr{E}(\mathbf{p}) \text { subject to } \mathbf{p} \geqslant \mathbf{0},
$$

where $\mathbf{p}$ is a vector of element properties, e.g., the axial rigidities, $\alpha_{i}$, and the mass density per unit length, $\rho_{i}$, and $\mathscr{E}$ is the error function.

There are two common ways of defining the error function: one is in terms of the difference between the computed and measured data, and the other is in terms of the unbalanced forces of the equilibrium equations. The latter definition is adopted here because no solution of the equilibrium equation is required in the formulation. This is important because the stiffness matrix in the frequency domain becomes singular 
at resonance frequencies. Unless special treatment is taken, such as incorporating artificial damping, no spectral response can be obtained at such frequencies. Therefore, the equation-error formulation is superior to the output-error formulation in this respect.

The error function adopted in this study is

$$
\mathscr{E}=\sum_{k=1}^{\mathscr{K}} \sum_{n=1}^{\mathcal{N}}\left|\hat{\mathbf{K}}\left(\omega_{n}\right) \hat{\mathbf{u}}_{k}\left(\omega_{n}\right)-\hat{\mathbf{F}}_{k}\left(\omega_{n}\right)\right|^{2}
$$

where $\mathscr{K}$ is the number of tests performed, $\mathscr{N}$ is the number of frequencies included, and $\hat{\mathbf{u}}_{k}$ and $\hat{\mathbf{F}}_{k}$ are the Fourier transforms of the measured response and nodal forces in the $k$ th test, respectively. The error function does not necessarily include all frequencies used in the discrete Fourier transform. However, a minimum number of spectral data are required in order to attain a solution. This issue will be discussed later.

For truss structures, each element is characterized by two parameters, namely $\alpha$ and $\rho$. Numerical experiments show that it is difficult to identify these two sets of parameters concurrently. This is because $\rho$ affects the governing equation only through the wavenumber $k_{n}=\omega_{n} \sqrt{\rho / \alpha}$. Obviously, the influence of $\rho$ is coupled with that of $\alpha$. Therefore, it is not easy to identify these two parameters simultaneously. Since the densities of truss members are often known with high confidence, they are assumed to be given in this study.

In real applications, it is usually difficult to measure all the nodal displacements. In general, the relation between the measured data and the nodal displacements in the frequency domain can be represented by

$$
\hat{\mathbf{Y}}\left(\omega_{n}\right)=\mathbf{C} \hat{\mathbf{u}}\left(\omega_{n}\right),
$$

where $\hat{\mathbf{Y}}\left(\omega_{n}\right)$, the Fourier transform of the measured data, is an $r \times 1$ vector, and $\mathbf{C}$ is an $r \times l$ constant matrix. If all the nodal displacements are measured, then $\mathbf{C}$ is simply the identity matrix $\mathbf{I}$.

Equation (10) can be rewritten as

$$
\hat{\mathbf{Y}}\left(\omega_{n}\right)=\left[\begin{array}{ll}
\mathbf{C}_{1} & \mathbf{C}_{2}
\end{array}\right]\left[\begin{array}{c}
\hat{\mathbf{u}}_{m}\left(\omega_{n}\right) \\
\hat{\mathbf{u}}_{u}\left(\omega_{n}\right)
\end{array}\right],
$$

where $\hat{\mathbf{u}}_{m}$ and $\hat{\mathbf{u}}_{u}$ are subvectors of $\hat{\mathbf{u}}$, corresponding to the measured and unmeasured nodal displacements, respectively; $\mathbf{C}$ is partitioned into two submatrices $\mathbf{C}_{1}$ and $\mathbf{C}_{2}$ such that $\mathbf{C}_{1}$ is an $r \times r$ non-singular matrix. For example, if parts of the nodal accelerations are measured in the test, then $\mathbf{C}_{1}=\omega_{n}^{2} \mathbf{I}$ and $\mathbf{C}_{2}=\mathbf{0}$.

Substituting equation (11) into the global equilibrium equation, one obtains

$$
\tilde{\mathbf{K}}\left(\omega_{n}\right) \hat{\mathbf{Y}}\left(\omega_{n}\right)=\tilde{\mathbf{F}}\left(\omega_{n}\right),
$$

where

$$
\tilde{\mathbf{K}}=\hat{\mathbf{K}}_{11} \mathbf{C}_{1}^{-1}-\left(\hat{\mathbf{K}}_{12}-\hat{\mathbf{K}}_{11} \mathbf{C}_{1}^{-1} \mathbf{C}_{2}\right)\left(\hat{\mathbf{K}}_{22}-\hat{\mathbf{K}}_{21} \mathbf{C}_{1}^{-1} \mathbf{C}_{2}\right)^{-1} \hat{\mathbf{K}}_{21} \mathbf{C}_{1}^{-1}
$$

and

$$
\tilde{\mathbf{F}}=\hat{\mathbf{F}}_{1}-\left(\hat{\mathbf{K}}_{12}-\hat{\mathbf{K}}_{11} \mathbf{C}_{1}^{-1} \mathbf{C}_{2}\right)\left(\hat{\mathbf{K}}_{22}-\hat{\mathbf{K}}_{21} \mathbf{C}_{1}^{-1} \mathbf{C}_{2}\right)^{-1} \hat{\mathbf{F}}_{2} .
$$

Hence, the error function can be defined as the norm of the residuals of equation (12).

There are various algorithms that can be used to solve the optimization problem (8). Based on past experience, the Broyden-Fletcher-Goldfarb-Shanno quasi-Newton method [25] is applied here because of the need for efficiency. In this method, it is necesary to 
compute the gradient of the error function with respect to the unknown parameters. If the nodal displacements are completely measured, it is easily derived that

$$
\nabla \mathscr{E}=2 \sum_{k=1}^{\mathscr{K}} \sum_{n=1}^{\mathscr{N}}\left[\hat{\mathbf{K}}\left(\omega_{n}\right) \hat{\mathbf{u}}_{k}\left(\omega_{n}\right)-\hat{\mathbf{F}}_{k}\left(\omega_{n}\right)\right]^{\mathrm{T}} \nabla \hat{\mathbf{K}}\left(\omega_{n}\right) \hat{\mathbf{u}}_{k}\left(\omega_{n}\right) .
$$

The gradient of global stiffness, $\nabla \hat{\mathbf{K}}$, can be obtained by assembling the gradient of the element stiffness, as follows:

$$
\frac{\partial \hat{\mathbf{k}}}{\partial \alpha}=\left[\begin{array}{cc}
\frac{k_{n} \sin k_{n} L \cos k_{n} L+k_{n}^{2} L}{2 \sin ^{2} k_{n} L} & \frac{-k_{n} \sin k_{n} L-k_{n}^{2} L \cos k_{n} L}{2 \sin ^{2} k_{n} L} \\
\frac{-k_{n} \sin k_{n} L-k_{n}^{2} L \cos k_{n} L}{2 \sin ^{2} k_{n} L} & \frac{k_{n} \sin k_{n} L \cos k_{n} L+k_{n}^{2} L}{2 \sin ^{2} k_{n} L}
\end{array}\right] .
$$

If the nodal displacements are only partially measured, a closed form expression for the gradient can be derived by using the relation $\nabla\left(\tilde{\mathbf{K}} \widetilde{\mathbf{K}}^{-1}\right)=\nabla \mathbf{I}=\mathbf{0}$.

\section{SUBSTRUCTURE IDENTIFICATION}

In the preceding section, a method for identifying a total truss was introduced. However, there are situations in which substructure identification is more appropriate: for instance, when a truss is damaged and one only wants to assess the element stiffness near the damaged location. The proposed identification method can be modified to meet this end.

First, divide the nodes into three groups: internal nodes, interface nodes and external nodes. Internal nodes are connected only to the elements of the substructure; interface nodes connect the substructure and the remaining part of the structure; all the remaining nodes are external nodes. According to such a partition, the force equilibrium at the internal nodes can be stated as

$$
\hat{\mathbf{K}}^{i s}\left(\omega_{n}\right) \hat{\mathbf{u}}^{s}\left(\omega_{n}\right)=\hat{\mathbf{F}}^{i}\left(\omega_{n}\right),
$$

where $\hat{\mathbf{K}}^{i s}\left(\omega_{n}\right)$ is a submatrix of $\hat{\mathbf{K}}\left(\omega_{n}\right), \hat{\mathbf{u}}^{s}\left(\omega_{n}\right)$ and $\hat{\mathbf{F}}^{i}\left(\omega_{n}\right)$ are subvectors of $\hat{\mathbf{u}}\left(\omega_{n}\right)$ and $\hat{\mathbf{F}}\left(\omega_{n}\right)$ respectively; the superscript $i$ denotes the rows corresponding to the internal nodes, and $s$ denotes the columns corresponding to the internal nodes as well as the interface nodes: in other words, all the nodes to which the substructure elements are connected.

Hence, identification of the substructure can be formulated as the optimization problem

$$
\operatorname{minimize} \quad \mathscr{E}_{s}=\sum_{k=1}^{\mathscr{K}} \sum_{n=1}^{\mathcal{N}}\left|\hat{\mathbf{K}}^{i s}\left(\omega_{n}\right) \hat{\mathbf{u}}_{k}^{s}\left(\omega_{n}\right)-\hat{\mathbf{F}}_{k}^{i}\left(\omega_{n}\right)\right|^{2},
$$

where $\mathscr{E}_{s}$ is the new error function for the substructure identification. Unlike equation (9), this error function is in terms of the residual forces at the internal nodes, not all nodes. Therefore, the loads must be applied at the internal nodes in at least one of the tests. Otherwise, $\hat{\mathbf{F}}_{k}^{i}=0$, and one arrives only at the trivial solution.

This optimization program has exactly the same structure as that in equation (9). Hence, the solution procedure is identical to that of total identification except that $\hat{\mathbf{K}}$, $\hat{\mathbf{u}}_{k}$, and $\hat{\mathbf{F}}_{k}$ are replaced by $\hat{\mathbf{K}}^{i s}, \hat{\mathbf{u}}_{k}^{s}$ and $\hat{\mathbf{F}}_{k}^{i}$, respectively.

\section{IDENTIFIABILITY}

Sanayei and Onipede [10] have discussed the relationship between identifiability and the number of independent measurements in the static identification. In their approach, only 
one degree of freedom (DOF) is loaded in each test, and the number of independent measurements is

$$
N I M=\left(m_{1}+m_{2}\right)\left(m_{2}+m_{3}\right)-m_{2}\left(m_{2}-1\right) / 2,
$$

where $m_{1}$ is the number of DOF's at which only force is applied, $m_{2}$ is the number of DOF's at which force is applied and the displacement is measured, and $m_{3}$ is the number of DOF's at which only the displacement is measured. In other words, $N I M$ is equal to the total number of measurements minus the number of redundant measurements according to Betti's reciprocal theorem. It is claimed [10] that a unique set of parameters can be identified if NIM is greater than or equal to the number of unknown parameters.

In this study, a similar but less restrictive formula is proposed for use in this application, namely

$$
N I M=(\mathscr{K} \mathscr{M}-\mathscr{B}) \mathscr{N},
$$

where $\mathscr{M}$ is the number of responses measured in each test, and $\mathscr{B}$ is the number of redundant measurements according to Betti's reciprocal theorem. As opposed to Sanayei and Onipede's formula, force can be applied to more than one DOF in each test. In the special case that only one DOF is loaded in each test, then $\mathscr{K}=m_{1}+m_{2}$, $\mathscr{M}=m_{2}+m_{3}$ and $\mathscr{B}=m_{2}\left(m_{2}-1\right) / 2$.

Notice that both equations (19) and (20) only set a lower bound for the number of measurements needed. There are situations in which there exist an infinite number of solutions even when NIM is greater than the number of unknown parameters. These solutions are called the output-equivalent solutions because the responses of all the corresponding trusses are consistent with the measured data. Therefore, it is advisable to take as many measurements as possible to avoid output-equivalent solutions.

\section{NOISE SIMULATION}

To investigate the influence of noise, it is a common practice to superpose white noise to the computed transient response of a structure to simulate the measurment errors. Then, the influence of noise is investigated by comparing the results obtained when using contaminated data and noise-free data, respectively. In this study, the response of the truss is computed in the frequency domain, not in the time domain. One cannot superpose white noise on the computed response directly. Therefore, it is necessary to establish the relation between the characteristics of the white noise in the time domain and in the frequency domain.

Let $r(t)$ and $\hat{r}\left(\omega_{n}\right)$ denote the noise in the time domain, respectively. $r(t)$ and $\hat{r}\left(\omega_{n}\right)$ are related by

$$
r(t)=\sum_{n=0}^{N-1} \hat{r}\left(\omega_{n}\right) \mathrm{e}^{\mathrm{i} \omega_{n} t}
$$

Assume that $r(t)$ is a white process with zero mean and standard deviation $\sigma$. Theoretically, the amplitude of $\hat{r}\left(\omega_{n}\right), A$, is constant in the frequency domain. The relation between $A$ and $\sigma$ can be obtained as follows. According to Parseval's theorem for the discrete Fourier transform [26],

$$
\sum_{i=0}^{N-1} r_{i}^{2}=\frac{1}{N} \sum_{n=0}^{N-1}\left|\hat{r}_{n}\right|^{2}
$$


where $r_{i}=r(i \Delta t)$ and $\hat{r}_{n}=\hat{r}\left(\omega_{n}\right)$. Dividing both sides of the above equation by $N$, one obtains

$$
\sigma^{2}=\frac{1}{N^{2}} \sum_{n=0}^{N-1}\left|\hat{r}_{n}\right|^{2}=\frac{A^{2}}{N}
$$

Hence,

$$
\sigma=A / \sqrt{N}
$$

In error analysis, $\sigma$ is often taken to be a fraction of the root mean square of the response of the structure. Applying Parseval's theorem again, one finds that the root mean square of $u(t)$ is

$$
R M S(u) \equiv \sqrt{\frac{1}{N} \sum_{i=0}^{N-1} u_{i}^{2}}=\sqrt{\frac{1}{N^{2}} \sum_{n=0}^{N-1}\left|\hat{u}_{n}\right|^{2}}
$$

If $\sigma$ is assumed to be $\zeta \%$ of $\operatorname{RMS}(u)$, then

$$
\sigma=(\zeta / 100) R M S(u)
$$

Subsituting equations (24) and (25) into equation (26), one obtains the amplitude of the noise in the frequency domain as

$$
A=\frac{\zeta}{100} \sqrt{\frac{1}{N} \sum_{n=0}^{N-1}\left|\hat{u}_{n}\right|^{2}} .
$$

This formula gives the amplitude of the random noise to be superposed on the response of the structure in the frequency domain to simulate the measurement errors.

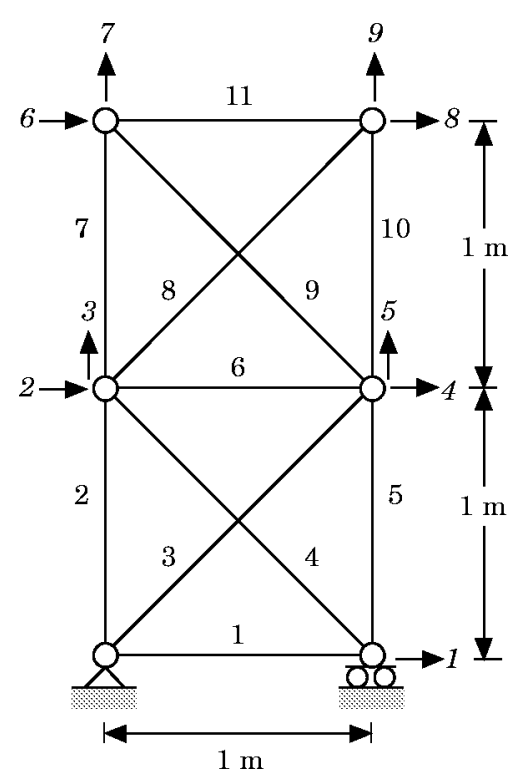

Figure 1. An example truss. 


\section{NUMERICAL EXAMPLE}

Consider a simply supported truss with 11 members and six nodes, as shown in Figure 1. The truss has nine DOF's. The members are made of aluminum with Young's modulus $70 \mathrm{GPa}$ and mass density $2700 \mathrm{~kg} / \mathrm{m}^{3}$. The cross-sectional area is $0.002 \mathrm{~m}^{2}$ for elements 1 to 5 and $0.001 \mathrm{~m}^{2}$ for the other elements. Hence, the initial axial rigidity and mass densities per unit length of the members are respectively $140 \mathrm{MN}$ and $5.4 \mathrm{~kg} / \mathrm{m}$ for elements 1 to 5 and $70 \mathrm{MN}$ and $2.7 \mathrm{~kg} / \mathrm{m}$ for the other elements. The axial rigidities are then reduced to the values shown in Table 1 to simulate the truss degradation after a period of service. To demonstrate better the effectiveness and efficiency of the proposed algorithm, the initial guess is set to be $200 \mathrm{MN}$ for elements 1 to 5 and $100 \mathrm{MN}$ for the other elements.

A numerical test was performed on a DEC 3000/4000 workstation. In the test, a half-sine shaped pulse as shown in Figure 2 was applied at DOF 8, and the displacements were computed by the spectral finite element method. 4096 data points with frequency spacing $(\Delta \omega) 50 \mathrm{rad} / \mathrm{s}$ were assumed to be collected. The spectral displacements of the truss are shown in Figure 3. The simulated results were then used as input to identify the properties of the truss.

First, consider the case in which all displacements are known. In this case, $\mathscr{K}=1$, $\mathscr{M}=9$ and $\mathscr{B}=0$. Since there are 11 unknowns, according to equation (20), spectral data corresponding to at least two frequencies are needed to determine the axial rigidities. The spectral data corresponding to $\omega=1000$ and $1050 \mathrm{rad} / \mathrm{s}$ were arbitrarily selected to identify the properties. Since there was no noise in the data, the identified $\alpha^{\prime}$ s were exact.

Next, consider the case is which only partial displacements are known. Suppose the displacements at DOF's 3, 4, 6, 7, 8 and 9 are measured. There are only six independent measurements but 11 unknowns. Apparently, at least two sets of spectral data are needed. Similarly, the spectral data corresponding to $\omega=1000$ and $1050 \mathrm{rad} / \mathrm{s}$ were selected to identify the properties, and the axial rigidities were identified successfully. The computation time (CPU) was $36.2 \mathrm{~s}$, which is 30 times longer than the first case. It seems that the more displacements are measured in each test, the faster the optimization process converges.

TABLE 1

$\alpha$ identified by using various spectral data-response at DOF's 3, 4, 6 and 9 measured

\begin{tabular}{cccccc}
\hline \multirow{2}{*}{ Element } & $\begin{array}{c}\text { Exact } \\
\text { value }\end{array}$ & $\begin{array}{c}\text { Initial } \\
\text { guess }\end{array}$ & $\begin{array}{c}\omega=1000-1100 \\
\Delta \omega=50\end{array}$ & $\begin{array}{c}\omega=1000-1200 \\
\Delta \omega=50\end{array}$ & $\omega=1600,3750,4050$ \\
\hline 1 & $126 \cdot 0$ & 200.0 & $123 \cdot 5$ & $126 \cdot 0$ & $126 \cdot 0$ \\
2 & $140 \cdot 0$ & $200 \cdot 0$ & $140 \cdot 2$ & $140 \cdot 0$ & $140 \cdot 0$ \\
3 & $133 \cdot 0$ & $200 \cdot 0$ & $132 \cdot 6$ & $133 \cdot 0$ & $133 \cdot 0$ \\
4 & $126 \cdot 0$ & $200 \cdot 0$ & $128 \cdot 2$ & $126 \cdot 0$ & $126 \cdot 0$ \\
5 & $133 \cdot 0$ & $200 \cdot 0$ & $132 \cdot 9$ & $133 \cdot 0$ & $73 \cdot 0$ \\
6 & $70 \cdot 0$ & $100 \cdot 0$ & $71 \cdot 7$ & $70 \cdot 0$ & $63 \cdot 0$ \\
7 & $63 \cdot 0$ & $100 \cdot 0$ & $63 \cdot 9$ & $63 \cdot 0$ & $66 \cdot 5$ \\
8 & $66 \cdot 5$ & $100 \cdot 0$ & $66 \cdot 3$ & $66 \cdot 5$ & $70 \cdot 0$ \\
9 & $70 \cdot 0$ & $100 \cdot 0$ & $69 \cdot 7$ & $70 \cdot 0$ & $63 \cdot 0$ \\
10 & $63 \cdot 0$ & $100 \cdot 0$ & $63 \cdot 0$ & $63 \cdot 0$ & $66 \cdot 5$ \\
11 & $65 \cdot 5$ & $100 \cdot 0$ & $66 \cdot 5$ & $66 \cdot 5$ & $57 \mathrm{~s}$ \\
\hline $\mathrm{CPU}$ & & & $3417 \mathrm{~s}$ & $3788 \mathrm{~s}$ & \\
\hline
\end{tabular}


The third case considered is that only the displacements at less than half of the DOF's are measured, namely DOF's 3, 4, 6 and 9. In this case, at least three sets of spectral data are required. Identification was carried out using three $(\omega=1000-1100 \mathrm{rad} / \mathrm{s})$ and five sets $(\omega=1000-1200 \mathrm{rad} / \mathrm{s})$ of spectral data at equally spaced frequencies, respectively. The results are shown in Table 1. The same conclusion can be drawn that the more spectral data are used, the better are the results obtained. The maximum error is $2.5 \%$ even when only three sets of spectral data are used. One can also see that the computation time increases with the number of data sets used.

Examination of Figure 3 shows that the response curves contain peaks near frequencies $\omega=1600,3750,4050 \mathrm{rad} / \mathrm{s}$, and so on. These are obviously the resonant frequencies of the truss. The results of identification by using the spectral data corresponding to $\omega=1600,3750$ and $4050 \mathrm{rad} / \mathrm{s}$ are also shown in Table 1 . Obviously, using only three sets of resonance data can achieve the same accuracy as using five sets of spectral data at equally spaced frequencies. This is mainly due to the strong response of the truss near resonance frequencies. Furthermore, convergence is achieved in only one sixtieth of the CPU time for the five set case. Hence, it is recommended that the spectral data near the resonance frequencies be used.

In real applications, noise is always present in the measured response. Hence, the influence of measurement error on the identified properties needs to be investigated. In the following, $10 \%$ random noise is superposed on the spectral response to simulate the measurement errors. For each of the cases presented, 100 Monte Carlo simulations were performed and the mean values and coefficients of variation (c.o.v.) of the $\alpha^{\prime}$ s were evaluated. The mean value of $\alpha$ is denoted $\bar{\alpha}$ hereafter.

First, consider the case in which all displacements are known. Again, the load was applied at DOF 8. As in the noise-free case, two sets of spectral data seem to be adequate according to equation (20). However, if spectral data at equally spaced frequencies
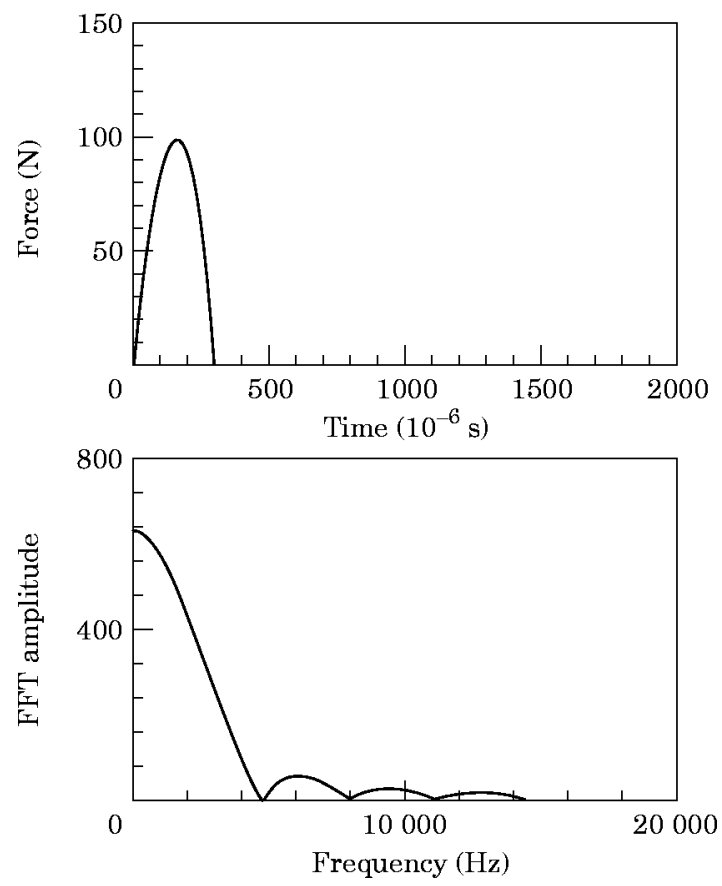

Figure 2. The input force for the numerical example. 
(a)
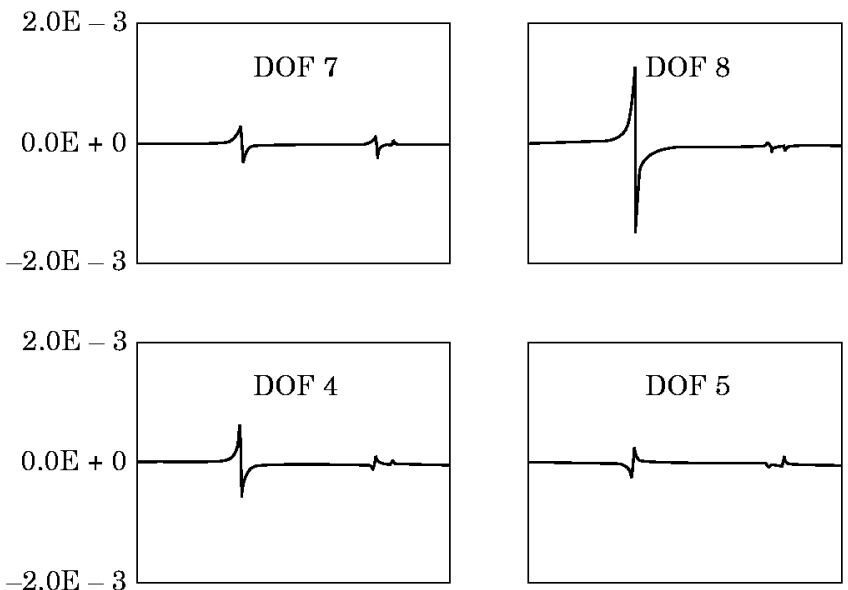

$-2.0 \mathrm{E}-3$
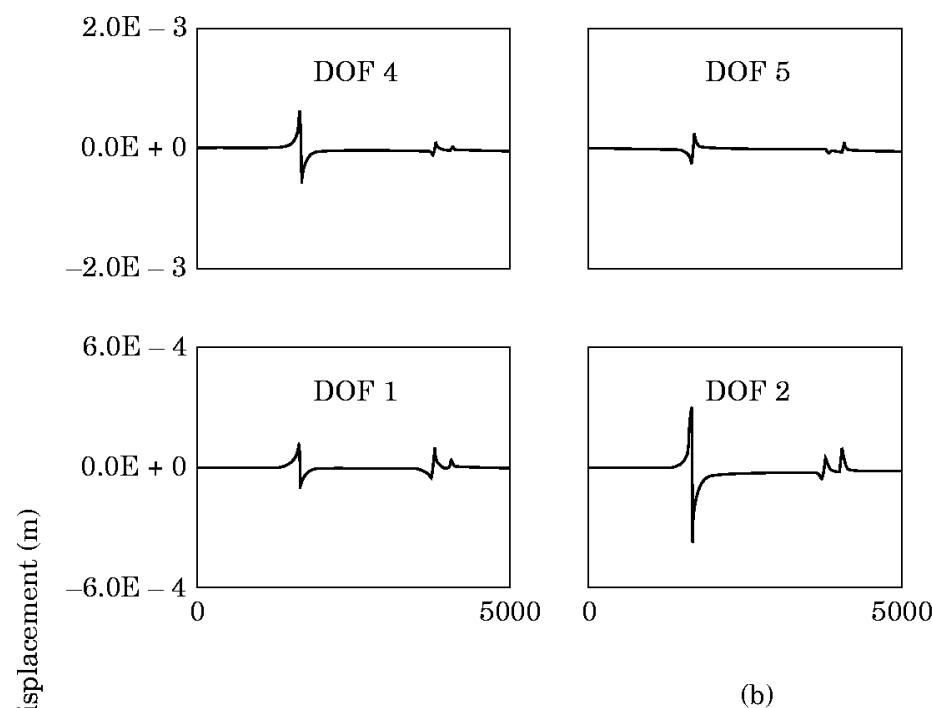

(b)
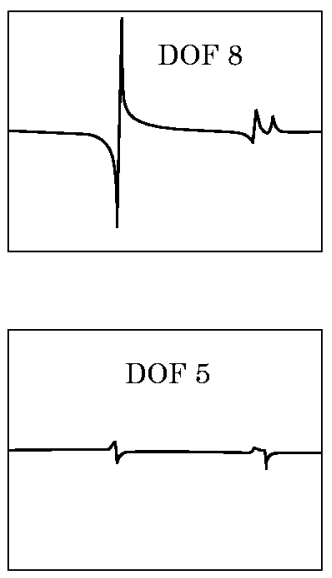

$-6.0 \mathrm{E}-4$
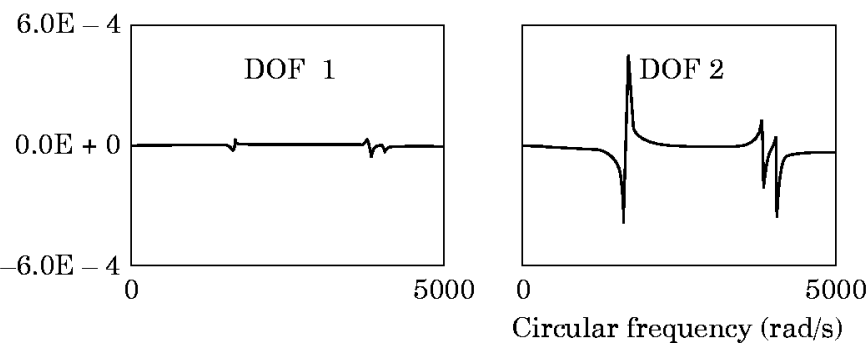
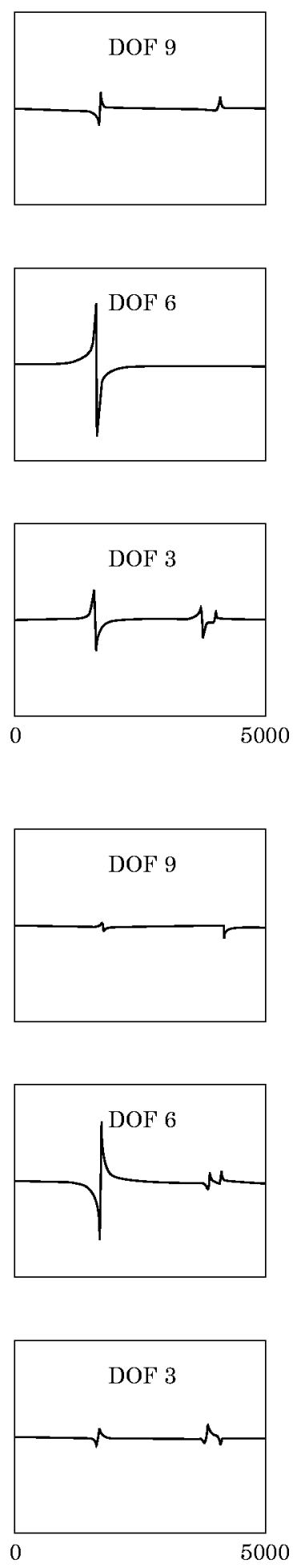

Figure 3. The spectral displacements due to an impulsive load at DOF 8: (a) real part; (b) imaginary part. 
was used, the identification failed even when 60 sets of data were employed. This is because the response of the truss is small off the resonance frequencies, and the magnitude of the noise may exceed that of the nodal displacements at non-resonance frequencies. Consequently, the data at such frequencies are corrupted by the presence of noise. To remedy this situation, one may use more spectral data. It was found that identification with 80 sets of spectral data was successful. The maximum error of the $\alpha^{\prime}$ s is $16.4 \%$ and the maximum c.o.v. of the $\alpha^{\prime} \mathrm{s}$ is $3 \cdot 1 \%$, as shown in Table 2 . The results can be further improved by performing more tests. It is shown in Table 2 that if an extra test is carried out with the load applied at DOF 9, the maximum error of the $\bar{\alpha}^{\prime}$ s reduces to $6 \cdot 2 \%$.

In fact, a more effective measure to deal with contaminated data is to use data near resonance frequencies. This is because the signal to noise ratio is increased by several orders of magnitude when the resonance data are used. The results obtained by using nine sets of spectral data $(\omega=1550,1600,1650,3700,3750,3800,400,4050$ and $4100 \mathrm{rad} / \mathrm{s})$ near the first three resonance frequencies are shown in Table 3 . These nine sets of resonance data are included in the aforementioned 80 sets of spectral data. It is seen that the mean values of the $\alpha^{\prime}$ s have improved significantly, although the c.o.v.'s are slightly higher than for the 80 -set cases. This implies that the off-resonance data have little contribution to the identification of element rigidities. In fact, the computation time is increased by an order of magnitude when such data are included. Table 3 also shows the results of a two-test case with loading applied at DOF's 8 and 9, respectively. It is obvious that the more tests performed, the better the results are.

In order to see how the identification results deteriorate as the noise level increases, the identification was performed for various noise levels. Two tests were performed with the loading being applied at DOF's 8 and 9, respectively. Nine sets of spectral data near resonance frequencies were employed to identify the $\alpha^{\prime}$ s. First, consider the case in which all displacements are measured. The relative error and c.o.v. of $\alpha_{11}$ under various noise levels are shown in Figure 4. Generally speaking, the c.o.v. increases linearly with the magnitude of the noise. The linear relationship between the c.o.v. of the identified rigidities and the noise level suggests that the proposed method is stable. This is an important feature because an identification method is of no practical use if the errors blow up quickly as the noise comes in.

TABLE 2

$\alpha$ identified by contaminated data response at all DOF's measured; $\omega=1000-4950, \Delta \omega=50$

\begin{tabular}{|c|c|c|c|c|}
\hline \multirow[b]{2}{*}{ Element } & \multicolumn{2}{|c|}{ One test } & \multicolumn{2}{|c|}{ Two tests } \\
\hline & $\bar{\alpha}$ & c.o.v. $(\%)$ & $\bar{\alpha}$ & c.o.v. $(\%)$ \\
\hline 1 & $122 \cdot 47$ & 0.6 & $123 \cdot 44$ & 0.4 \\
\hline 2 & $133 \cdot 42$ & 0.5 & 135.92 & $0 \cdot 3$ \\
\hline 3 & $128 \cdot 41$ & 0.8 & $132 \cdot 77$ & $0 \cdot 4$ \\
\hline 4 & $121 \cdot 75$ & 1.0 & $122 \cdot 03$ & $1 \cdot 0$ \\
\hline 5 & $127 \cdot 32$ & 0.4 & $132 \cdot 34$ & 0.3 \\
\hline 6 & $64 \cdot 86$ & $2 \cdot 0$ & $70 \cdot 00$ & 0.5 \\
\hline 7 & $57 \cdot 12$ & $1 \cdot 3$ & $59 \cdot 10$ & $1 \cdot 0$ \\
\hline 8 & $63 \cdot 57$ & $1 \cdot 0$ & $65 \cdot 11$ & 1.0 \\
\hline 9 & $66 \cdot 54$ & $1 \cdot 0$ & $69 \cdot 75$ & 0.6 \\
\hline 10 & $58 \cdot 25$ & $1 \cdot 3$ & $62 \cdot 48$ & 0.2 \\
\hline 11 & $55 \cdot 57$ & $3 \cdot 1$ & $64 \cdot 03$ & 0.9 \\
\hline $\mathrm{CPU}$ & \multicolumn{2}{|c|}{$36 \cdot 4 \mathrm{~s}$} & \multicolumn{2}{|c|}{$60 \cdot 8 \mathrm{~s}$} \\
\hline
\end{tabular}


TABLE 3

a identified by using contaminated data-response at all DOF's measured; $\omega=1550,1600,1650,3700,3750,3800,4000,4050,4100$

\begin{tabular}{|c|c|c|c|c|}
\hline \multirow[b]{2}{*}{ Element } & \multicolumn{2}{|c|}{ One test } & \multicolumn{2}{|c|}{ Two tests } \\
\hline & $\bar{\alpha}$ & c.o.v. $(\%)$ & $\bar{\alpha}$ & c.o.v. $(\%)$ \\
\hline 1 & $125 \cdot 42$ & $0 \cdot 7$ & $125 \cdot 76$ & $0 \cdot 5$ \\
\hline 2 & $139 \cdot 25$ & $0 \cdot 5$ & $139 \cdot 52$ & $0 \cdot 4$ \\
\hline 3 & $132 \cdot 49$ & $0 \cdot 8$ & $132 \cdot 92$ & $0 \cdot 5$ \\
\hline 4 & $125 \cdot 58$ & $1 \cdot 0$ & $125 \cdot 74$ & $1 \cdot 2$ \\
\hline 5 & $132 \cdot 32$ & $0 \cdot 4$ & $132 \cdot 96$ & $0 \cdot 3$ \\
\hline 6 & $69 \cdot 51$ & $2 \cdot 3$ & $70 \cdot 00$ & $0 \cdot 6$ \\
\hline 7 & $62 \cdot 26$ & $1 \cdot 3$ & $62 \cdot 49$ & $1 \cdot 0$ \\
\hline 8 & $66 \cdot 29$ & $1 \cdot 1$ & $66 \cdot 36$ & $1 \cdot 1$ \\
\hline 9 & $69 \cdot 54$ & 0.9 & $70 \cdot 00$ & $0 \cdot 5$ \\
\hline 10 & $62 \cdot 45$ & $1 \cdot 1$ & $62 \cdot 93$ & $0 \cdot 2$ \\
\hline 11 & $64 \cdot 04$ & $4 \cdot 4$ & $66 \cdot 10$ & $1 \cdot 1$ \\
\hline CPU & \multicolumn{2}{|c|}{$6 \cdot 1 \mathrm{~s}$} & \multicolumn{2}{|c|}{$8 \cdot 2 \mathrm{~s}$} \\
\hline
\end{tabular}

Next, consider the cases in which only four and six displacements are measured. The results are listed in Table 4. It is seen that the c.o.v. of $\alpha$ and the CPU time decrease as the number of displacements measured increase. It seems that the more displacements are measured in each test, the less the results are influenced by the noise. Therefore, it is advisable to measure as many displacements as possible in each test.

The effectiveness of the proposed method in reconstruction of the properties of a damaged truss also has been investigated. Suppose the truss is damaged at element 7, and the axial rigidity of this element is reduced to $50 \%$ of the original value, i.e., $35 \mathrm{MN}$. Two tests were performed, the load being applied at DOF's 8 and 9, respectively. Nine sets of contaminated $(\zeta=10 \%)$ data $(\omega=1550,1600,1650,3550,3600,3650,4000,4050$ and $4100 \mathrm{rad} / \mathrm{s}$ ) near the resonance frequencies were employed to identify the $\alpha$ 's. Two
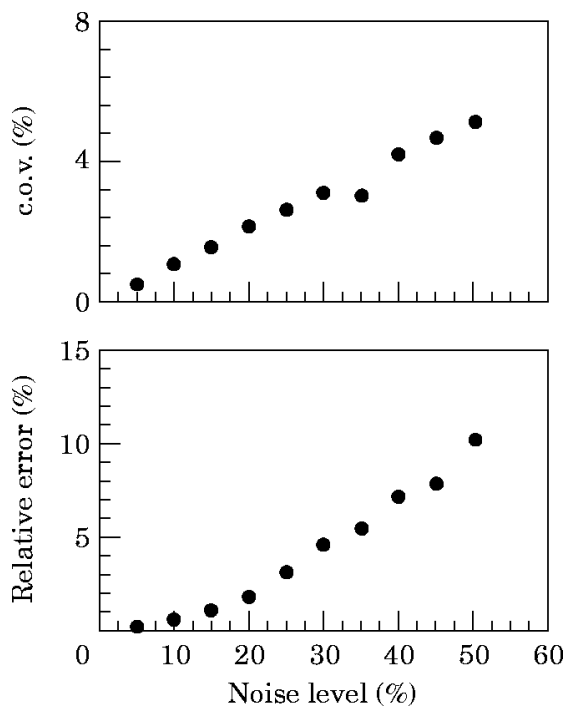

Figure 4. The identification result of element 11. 
TABLE 4

$\alpha$ identified by using contaminated data- $\omega=1550,1600,1650,3700$, $3750,3800,4000,4050,4100$

\begin{tabular}{|c|c|c|c|c|}
\hline \multirow[b]{3}{*}{ Element } & \multicolumn{4}{|c|}{ Measured DOF's } \\
\hline & \multicolumn{2}{|c|}{$3,4,6,7,8,9$} & \multicolumn{2}{|c|}{$3,4,6,9$} \\
\hline & $\bar{\alpha}$ & c.o.v. $(\%)$ & $\bar{\alpha}$ & c.o.v. $(\%)$ \\
\hline 1 & $126 \cdot 36$ & $2 \cdot 1$ & $135 \cdot 60$ & $5 \cdot 6$ \\
\hline 2 & $139 \cdot 47$ & $0 \cdot 4$ & $139 \cdot 20$ & 0.7 \\
\hline 3 & $133 \cdot 33$ & $0 \cdot 5$ & 132.08 & $0 \cdot 9$ \\
\hline 4 & $124 \cdot 57$ & $3 \cdot 0$ & $118 \cdot 37$ & $5 \cdot 3$ \\
\hline 5 & $134 \cdot 12$ & $0 \cdot 9$ & $137 \cdot 40$ & $2 \cdot 1$ \\
\hline 6 & $70 \cdot 78$ & $0 \cdot 8$ & $68 \cdot 77$ & $3 \cdot 3$ \\
\hline 7 & $62 \cdot 07$ & $1 \cdot 0$ & $61 \cdot 12$ & $2 \cdot 7$ \\
\hline 8 & $66 \cdot 83$ & $1 \cdot 3$ & $69 \cdot 29$ & $3 \cdot 7$ \\
\hline 9 & $69 \cdot 50$ & $1 \cdot 2$ & $67 \cdot 58$ & $3 \cdot 1$ \\
\hline 10 & 61.94 & $1 \cdot 5$ & $60 \cdot 79$ & $3 \cdot 3$ \\
\hline 11 & $65 \cdot 03$ & $1 \cdot 6$ & $72 \cdot 25$ & $10 \cdot 8$ \\
\hline $\mathrm{CPU}$ & \multicolumn{2}{|c|}{$256.4 \mathrm{~s}$} & \multicolumn{2}{|c|}{$455 \cdot 5 \mathrm{~s}$} \\
\hline
\end{tabular}

cases were investigated: in case 1, six displacements were measured; in case 2, four displacements were measured. The results are listed in Table 5. It is seen that the damaged member can be identified successfully. The maximum errors of $\bar{\alpha}$ are $6 \cdot 1 \%$ and $5.4 \%$ for the 6-DOF case and 4-DOF case, respectively. Notice that the identification errors are of the same magnitude as the input error. This indicates again that the proposed method is stable.

Finally, substructure identification was conducted for both the original and damaged trusses. It was assumed that element 7, 8, 9, 10 and 11 are the target elements, and thus nodes 5 and 6 were the internal nodes. Two tests were performed, the loading being applied at DOF's 8 and 9 , respectively. Nine sets of contaminated data $(\zeta=10 \%)$ near the

TABLE 5

$\alpha$ identified for the damaged truss by using contaminated data$\omega=1550,1600,1650,3550,3600,3650,4000,4050,4100$

\begin{tabular}{|c|c|c|c|c|}
\hline \multirow[b]{3}{*}{ Element } & \multicolumn{4}{|c|}{ Measured DOF's } \\
\hline & \multicolumn{2}{|c|}{$3,4,6,7,8,9$} & \multicolumn{2}{|c|}{$3,4,6,9$} \\
\hline & $\bar{\alpha}$ & c.o.v. $(\%)$ & $\bar{\alpha}$ & c.o.v. $(\%)$ \\
\hline 1 & $126 \cdot 27$ & $2 \cdot 5$ & $132 \cdot 86$ & $5 \cdot 5$ \\
\hline 2 & $139 \cdot 70$ & $0 \cdot 4$ & $139 \cdot 24$ & $0 \cdot 6$ \\
\hline 3 & $132 \cdot 94$ & $0 \cdot 5$ & $132 \cdot 76$ & $0 \cdot 7$ \\
\hline 4 & $125 \cdot 23$ & $3 \cdot 5$ & $120 \cdot 82$ & $5 \cdot 1$ \\
\hline 5 & $134 \cdot 22$ & $1 \cdot 0$ & $135 \cdot 64$ & $1 \cdot 7$ \\
\hline 6 & $71 \cdot 53$ & $1 \cdot 4$ & $69 \cdot 79$ & $2 \cdot 1$ \\
\hline 7 & $34 \cdot 82$ & $0 \cdot 7$ & $34 \cdot 79$ & $0 \cdot 9$ \\
\hline 8 & $66 \cdot 48$ & $1 \cdot 3$ & $66 \cdot 87$ & $2 \cdot 0$ \\
\hline 9 & $69 \cdot 60$ & $1 \cdot 3$ & $69 \cdot 26$ & $2 \cdot 6$ \\
\hline 10 & $61 \cdot 69$ & $1 \cdot 6$ & $60 \cdot 99$ & $2 \cdot 8$ \\
\hline 11 & $62 \cdot 45$ & $2 \cdot 4$ & $68 \cdot 67$ & $10 \cdot 0$ \\
\hline
\end{tabular}


TABLE 6

Results of substructure identification when using contaminated data

\begin{tabular}{cccc}
\hline Element & Exact value & $\bar{\alpha}$ & c.o.v. $(\%)$ \\
\hline Original truss & & & \\
7 & $63 \cdot 00$ & $62 \cdot 99$ & $0 \cdot 8$ \\
8 & $66 \cdot 50$ & $66 \cdot 57$ & $0 \cdot 9$ \\
9 & $70 \cdot 00$ & $69 \cdot 96$ & $0 \cdot 5$ \\
10 & $63 \cdot 00$ & $63 \cdot 00$ & $0 \cdot 2$ \\
11 & $66 \cdot 50$ & $66 \cdot 30$ & $1 \cdot 0$ \\
Damaged truss & & & \\
7 & $35 \cdot 00$ & $37 \cdot 01$ & $0 \cdot 6$ \\
8 & $66 \cdot 50$ & $67 \cdot 05$ & $1 \cdot 0$ \\
9 & $70 \cdot 00$ & $71 \cdot 21$ & $0 \cdot 7$ \\
10 & $63 \cdot 00$ & $63 \cdot 06$ & $0 \cdot 4$ \\
11 & $66 \cdot 50$ & $63 \cdot 69$ & $2 \cdot 3$ \\
\hline
\end{tabular}

resonance frequencies were employed. The identified rigidities of the substructure elements are shown in Table 6 . The results are very close to those of the total identification for both the original and damaged trusses.

\section{CONCLUSIONS}

An algorithm to determine the element properties of truss structures by using their transient responses has been presented. The identification problem is formulated as an optimization program in which the error norm of the unbalanced nodal forces in the frequency domain is minimized. The spectral finite element method is employed to formulate the governing equations of the truss structures.

The numerical example demonstrates the effectiveness of the proposed algorithm to identify the element properties of a truss. Some conclusions can be drawn from the example. First, the more independent measurements are made, the more reliable the results are. Second, the spectral data near the resonance frequencies should be used in the identification because of accuracy and efficiency concerns. Third, the accuracy and efficiency of the identification process improves as the number of displacements measured in each test increases. Fourth, the proposed method is very stable. The magnitude of the identification errors is the same as that of the input errors. Finally, the element rigidities can be recovered successfully whether in total identification or in substructure identification. Therefore, the proposed method can serve as a non-destructive test to evaluate the safety of a truss because it can detect the damaged location of a truss precisely.

It should be mentioned that what is solved in this study is really a model problem. Identification of real structures is a much more complicated problem. For example, a real structure is seldom strictly undamped, and the noise may not be modeled as additive white noise. Such factors should be studied in future research.

\section{REFERENCES}

1. J. C. Chen and J. A. Gaba 1987 Proceedings of the AIAA/ASME/ASCE/AHS/ACE 28th Structures, Structural Dynamics and Materials Conference, New York. On orbit damage assessment for large space structures.

2. M. BARUCH and I. Y. BAR-ITZHACK 1978 American Institute of Aeronautics and Astronautics Journal 16(4), 346-351. Optimal weighted orthogonalization of measured modes. 
3. A. Berman and E. J. Nagy 1983 American Institute of Aeronautics and Astronautics Journal 21(8), 1168-1173. Improvement of a large analytical model using test data.

4. A. M. KaBE 1991 American Institute of Aeronautics and Astronautics Journal 23(9), 1431-1436. Stiffness matrix adjustment using mode data.

5. D. C. Kammer 1988 American Institute of Aeronautics and Astronautics Journal 26(1), 104-112. Optimal approximation for residual stiffness in linear system identification.

6. S. W. Smith and C. A. Beattie 1991 American Institute of Aeronautics and Astronautics Journal 29(1), 119-126. Secant-method adjustment using modal data.

7. C. Minas and D. J. Inman 1990 Journal of Vibration and Acoustics Transactions of the American Society of Mechanical Engineers, 112, 84-92. Matching finite element models to modal data.

8. D. C. Zimmerman and M. Kaouk 1992 American Institute of Aeronautics and Astronautics Journal 30(7), 1848-1855. Eigenstructure assignment approach for structural damage detection.

9. P.-L. Liu 1995 Journal of Structural Engineering, American Society of Civil Engineers 121(4), 599-608. Identification and damage detection of trusses using modal data.

10. M. SANAYEI and O. ONIPEDE 1991 American Institute of Aeronautics and Astronautics Journal 29(7), 1174-1179. Damage assessment of structures using static test data.

11. M. Sanayei and S. F. Scampoli 1991 Journal of Engineering Mechanics, American Society of Civil Engineers 117(5), 1021-1036. Structural element stiffness identification from static test data.

12. P. Hajela and F. J. Soerro 1990 American Institute of Aeronautics and Astronautics Journal 28(6), 1110-1115. Structural damage detection based on static and modal analysis.

13. P.-L. LiU and C.-C. Chian 1994 Proceedings, The 1994 Far East Conference on Nondestructive Testing, Taipei. Parametric identification of truss structures using static strains.

14. M. Shinozuka, C. B. Yun and H. Imai 1982 Journal of Engineering Mechanics, American Society of Civil Engineers 108(6), 1371-1390. Identification of linear structural dynamic systems.

15. M. S. Agbabian, S. F. Masri, R. K. Miller and T. K. Caughey 1991 Journal of Engineering mechanics 117(2), 370-390. System identification approach to detection of structural changes.

16. S. F. Masri, R. K. Miller, A. F. SAud and T. K. Caughey 1987 Journal of Aplied Mechanics 54, 918-922. Identification of nonlinear vibrating structures: part 1-formulation.

17. S. F. Masri, R. K. Miller, A. F. SAud and T. K. Caughey 1987 Journal of Applied Mechanics 54, 923-929. Identification of nonlinear vibrating structures: part 2-application.

18. C. C. Lin, T. T. Soong and H. G. Natke 1990 Journal of Engineering Mechanics, American Society of Civil Engineers 116(10), 2258-2274. Real-time system identification of degrading structures.

19. C. B. YUn and M. Shinozuka 1980 Journal of Structural Engineering, American Society of Civil Engineers 8(2), 187-203. Identification of nonlinear structural dynamic systems.

20. M. Hoshiya and E. Saito 1984 Journal of Engineering Mechanics, American Society of Civil Engineers 110(12), 1757-1770. Structural identification by extended Kalman filter.

21. M. Hoshiya and O. Maruyama 1991 Journal of Engineering Mechanics, American Society of Civil Engineers 117(7), 1442-1454. Adaptive identification of autoregressive processes.

22. J. E. Mottershead 1988 Journal of Vibration, Acoustics, Stress, and Reliability in Design 110, 360-365. A unified theory of recursive, frequency domain filters with application to system identification in structural dynamics.

23. J. F. Doyle 1989 Wave Propagation in Structures. New York: Springer-Verlag.

24. J. F. DoYle and T. N. FARris 1989 Computational Techniques for Impact, Penetration, and Perforation of Solids AMD-103, 9-29. Spectral analysis of impact induced wave propagation in 3-D frames.

25. D. G. Luenberger 1984 Linear and Nonlinear Programming. Reading, Massachusetts: AddisonWesley.

26. A. V. Oppenheim and R. W. Schafer 1989 Discrete-time signal Processing. Englewood Cliffs, New Jersey: Prentice-Hall. 